\title{
The Mobile Robot HILARE: Dynamic Modeling and Motion Simulation
}

\author{
M. Talezadeh, M. Ghazal, M. Nazemi-Zade, M. Taheri \\ Department of Mechanics, Damavand Branch, Islamic Azad University, Damavand, Iran
}

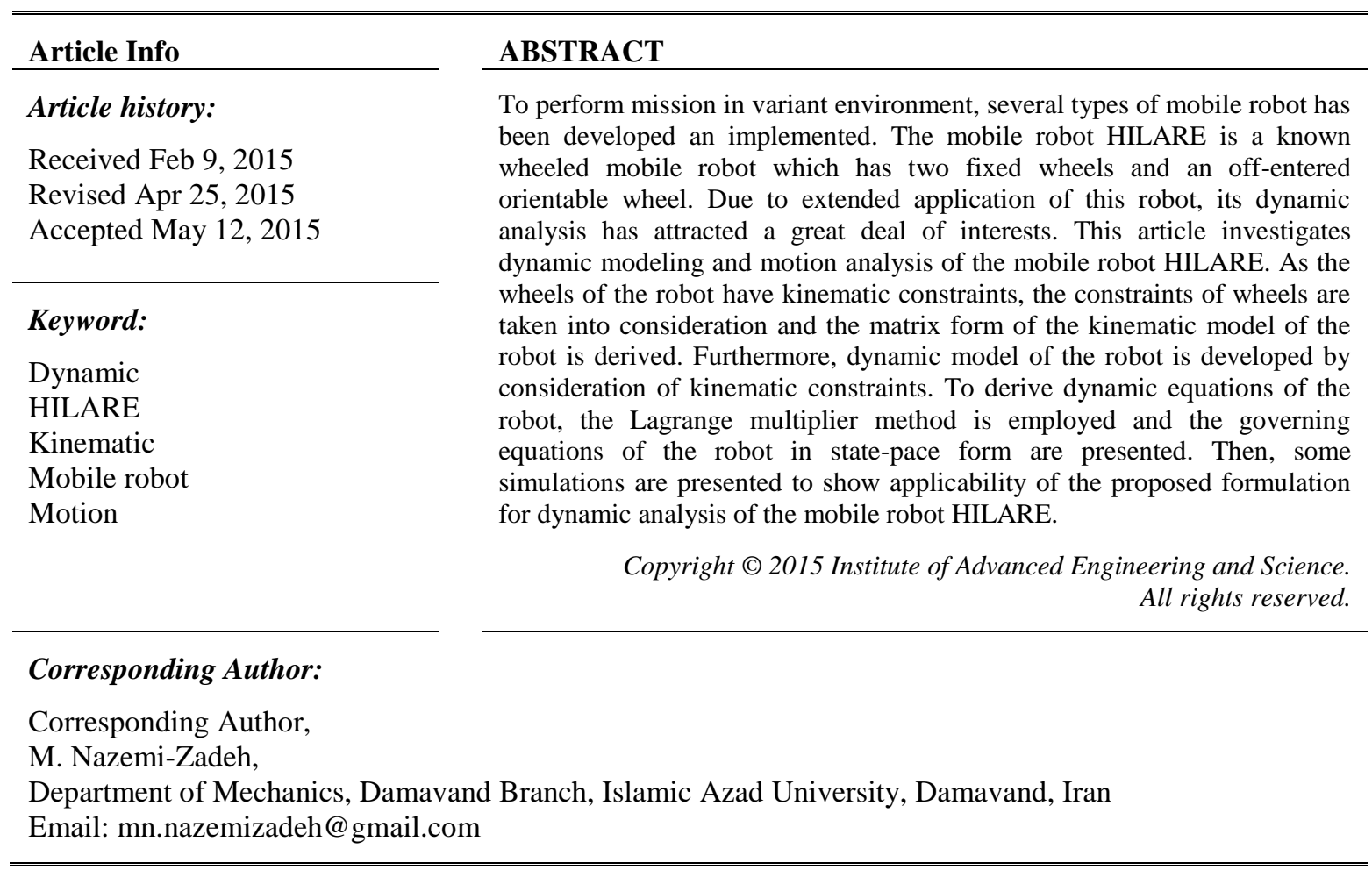

\section{INTRODUCTION}

Wheeled mobile robots are implemented in many applications due to their ability to operate in wide environments and extended surroundings. Despite of robots with fixed base, the mobile robots can move and convey loads from a first position to final one which are enough far from each other [1-6]. To perform mission in variant environment, several types of mobile robot has been developed an implemented. The mobile robot HILARE is a known wheeled mobile robot which has two fixed wheels and an off-entered orientable wheel. Due to extended application of this robot, its dynamic analysis has attracted a great deal of interests.

Generally, the mobile platform systems are distinguished by nonholonomic limitations. The nonholonomic constraints are known as kinematic non-integrable ones which cannot be eliminated from the dynamic model [5-8]. Those non-integrable limitations are offered as the wheels can only move in the direction of its plane and wheels must have pure rolling during the motion. The nonholonomic limitations make dynamic modeling of the wheeled mobile robot as an important and exigent problem. Thus, dynamic analysis of such systems has obtained numerous attractions by researchers. Some scientific authors studied the dynamic modeling of differential wheeled mobile platforms considering kinematic model of the system $[9,10]$. But, kinematic modeling has caused some troubles in applications, because actuator capacity and constraint of the mobile robot had not been taken into account. Chen et al. [11] presented path generation of wheeled mobile robot considering minimum time of travel. Nazemizadeh et al. [12] studied dynamic modeling and path control of a differential wheeled mobile robot based on optimal control method. Hence, in order to present the dynamic model of the system, they used an advanced nonlinear method to control path of a mobile robot which may encounter complication and numerical explosions. 
In this article, dynamic modeling of the mobile robot HILARE is presented. The nonholonomic constraints of its two fixed and one off-entered orientable wheel is expressed in the matrix form. Furthermore, dynamic model of the robot is developed by consideration of kinematic constraints. To derive dynamic equations of the robot, the Lagrange multiplier method is employed and the governing equations of the robot in state-pace form are presented. Then, some simulations are presented to show applicability of the proposed formulation for dynamic analysis of the mobile robot HILARE.

\section{DYNAMIC MODEL OF THE ROBOT}

Consider a mobile robot known as HILARE. As it is seen in figure (1), it has two fixed wheels and an off-centered wheel.

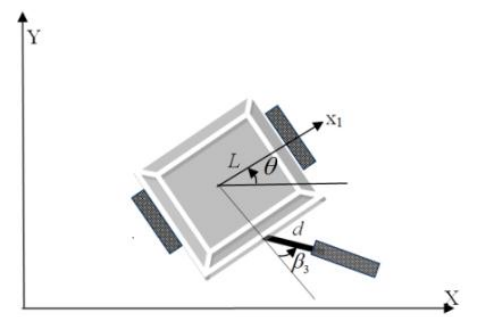

Figure 1. The mobile robot HILARE

As the dynamic equations of the robot are developed by Lagrange multiplier method, the total kinetic energy $(T)$ and potential energy (U) of the system must be computed. Then, by constructing the Lagrangian function $(L=T-U)$ and following the Lagrangian approach nonlinear equations of motion can be obtained. The Lagrangian equation can be formed as:

$$
\frac{d}{d t}\left(\frac{\partial L}{\partial \dot{q}_{i}}\right)-\frac{\partial L}{\partial q_{i}}=Q_{i}-\sum_{j=1}^{6} \lambda_{j} D_{j i}
$$

where $Q_{i}$ is the generalized force related to the generalize coordinate $q_{i}$, and $\lambda_{j}$ is unknown force related to each nonholonomic constraint of mobile base. Now, using Lagrangian equation the dynamic equations of the system can be obtained in the compact form as:

$$
M \ddot{\vec{q}}+\vec{V}(\vec{q}, \dot{\vec{q}})=B \vec{\tau}-A^{T} \vec{\lambda}
$$

in which $\vec{\tau} \in R^{n}$ is torque vector exerted to the joints, $M(\vec{q}) \in R^{n \times n}$ is the inertia matrix, $B$ is constant input matrix, $\vec{V}(\vec{q}, \dot{\vec{q}}) \in R^{n}$ is a vector which presents coriolis and gravitational forces and $A$ is a matrix which represents the kinematic constraints of the robot HILARE related to nonholonomic constraints of wheels.

Moreover, to eliminate the unknown forces from the dynamic equation of the system, the matrix $S$ is defined in such a form that $A S=0$. Therefore, the state-space presentation of the system represents a set of first-order differential equations as the following:

$$
\dot{\vec{x}}=\left[\begin{array}{c}
\dot{\vec{x}}_{1} \\
\dot{\vec{x}}_{2}
\end{array}\right]=\left[\begin{array}{c}
S v \\
f_{2}
\end{array}\right]+\left[\begin{array}{c}
0 \\
\left(S^{T} M S\right)^{-1}
\end{array}\right] \tau
$$

\section{DYNAMIC MODEL OF THE ROBOT}

In this section, the kinematic model of the mobile robot HILARE is presented. The mobile robot has two fixed wheels and a off-centered orientable wheel (Fig. 1). The parameters of robots are presented: $\beta_{3}$ is the angle of rotation of off-centered orientable wheel about the vertical axis, $d$ is the off-center length of the 
wheel, $C$ is the mass center of the mobile platform, $\theta$ is the heading angle of the mobile robot, $\phi_{i}$ is the rotation of the $\mathrm{i}^{\text {th }}$ wheel around its axle, $L$ is the distance from $C$ to the center point of the wheel. Also, the angle $\alpha$ is defined as the angle between the axle $x_{1}$ and a line from the center of each wheel to mass center $C$. One can consider that for the right fixed wheel $\alpha=0$ and for the fixed wheel.

Furthermore, each wheel of the HILARE has the following nonholonomic constraints: Each wheel can only move in the direction of its plane. Each wheel must have pure rolling without any slippage. Thus the mobile robot has six nonholonomic constraints regarding to their wheels which is expressed as:

$$
\begin{aligned}
& K T \dot{\vec{\psi}}+\Gamma \dot{\vec{\phi}}=0 \\
& \Omega T \dot{\vec{\psi}}+\dot{\beta}_{3} \vec{\chi}=0
\end{aligned}
$$

where $\vec{\psi}=\left[\begin{array}{lll}x_{c} & y_{c} & \theta\end{array}\right]^{T}$ is the mobile base posture vector, $\vec{\phi}=\left[\begin{array}{lll}\phi_{1} & \phi_{2} & \phi_{3}\end{array}\right]^{T}$ is the rotation vector of the wheels around its axle, $\vec{\chi}=\left[\begin{array}{lll}0 & 0 & d\end{array}\right]^{T}, T$ is the rotation matrix and $\Gamma$ is a diagonal matrix of radii of wheels:

$$
\begin{gathered}
T=\left[\begin{array}{ccc}
\cos \theta & \sin \theta & 0 \\
-\sin \theta & \cos \theta & 0 \\
0 & 0 & 1
\end{array}\right] \\
\Gamma=\left[\begin{array}{lll}
r & 0 & 0 \\
0 & r & 0 \\
0 & 0 & r
\end{array}\right]
\end{gathered}
$$

where $r$ is radius of each wheel of the mobile platform. Furthermore, each row of matrices $K$ and $\Omega$ represent the pure rolling and non lateral slippage, respectively. Thus the matrices are expressed as:

$$
\begin{aligned}
K & =\left[\begin{array}{ccc}
0 & 1 & L \\
0 & -1 & L \\
\cos \beta_{3} & \sin \beta_{3} & L \cos \beta_{3}
\end{array}\right] \\
\Omega & =\left[\begin{array}{ccc}
1 & 0 & 0 \\
-1 & 0 & 0 \\
\sin \beta_{3} & -\cos \beta_{3} & d+L \sin \beta_{3}
\end{array}\right]
\end{aligned}
$$

Now, to present the total kinematic constraints of the HILARE, the generalized coordinates of the two-wheeled mobile robot is defined as $\vec{q}=\left[\begin{array}{lllllll}x_{c} & y_{c} & \theta & \beta_{3} & \phi_{1} & \phi_{2} & \phi_{3}\end{array}\right]^{T}$ and the nonholonomic constraints of the system can be rewritten as:

$$
A(\vec{q}) \dot{\vec{q}}=0
$$
to:

Where the jacobian matrix $A$ represents the nonholonomic constraints of the system and it is equal

$$
A=\left[\begin{array}{lll}
K T & 0 & \Gamma \\
\Omega T & \vec{\chi} & 0
\end{array}\right]
$$


Therefore, matrices $A$ and $S$ which must be determined to derive the dynamic equations of the robot can be rewritten as:

$$
A=\left[\begin{array}{ccccccc}
-\sin \theta & \cos \theta & L & 0 & r & 0 & 0 \\
\sin \theta & -\cos \theta & L & 0 & 0 & r & 0 \\
\cos \left(\theta+\beta_{3}\right) & \sin \left(\theta+\beta_{3}\right) & L \cos \beta_{3} & 0 & 0 & 0 & r \\
\cos \theta & \sin \theta & 0 & 0 & 0 & 0 & 0 \\
-\cos \theta & -\sin \theta & 0 & 0 & 0 & 0 & 0 \\
\sin \left(\theta+\beta_{3}\right) & -\cos \left(\theta+\beta_{3}\right) & d+L \sin \beta_{3} & d & 0 & 0 & 0
\end{array}\right]
$$

$$
S=\left[\begin{array}{cc}
-\sin \theta & 0 \\
\cos \theta & 0 \\
0 & 1 \\
\frac{1}{d} \cos \beta_{3} & \frac{1}{d}\left(-d-L \sin \beta_{3}\right) \\
-\frac{1}{r} & -\frac{L}{r} \\
\frac{1}{r} & -\frac{L}{r} \\
-\frac{1}{r} \sin \beta_{3} & -\frac{L}{r} \cos \beta_{3}
\end{array}\right]
$$

\section{SIMULATION RESULTS}

In this section, dynamic motion of the mobile robot HILARE is simulated. The parameter of the robot is given as: $m_{c}$ is the mass of the mobile platform, $I_{c}$ is the moment of inertia of the mobile platform, $m_{w}$ is the mass of each wheel and $I_{w}$ is the moment of inertia of each wheel about its axis.

Furthermore, the values of the parameters are given as: $m_{c}=94 \mathrm{~kg}, m_{w}=5.2 \mathrm{~kg}, m_{w}=5.2 \mathrm{~kg}$, $m_{w}=5.2 \mathrm{~kg}, r=0.08 \mathrm{~m}, L=0.13 \mathrm{~m}$ and $d=0.1 \mathrm{~m}$. To simulate the path generation of the mobile platform, it is assumed that the robot moves from initial position $\left(\mathrm{x}_{\mathrm{c}}=0 \mathrm{~m}, \mathrm{y}_{\mathrm{c}}=0 \mathrm{~m}, \theta=0 \mathrm{rad}\right)$ to final position $\left(\mathrm{x}_{\mathrm{c}}=1.5 \mathrm{~m}, \mathrm{y}_{\mathrm{c}}=-0.4 \mathrm{~m}, \theta=0 \mathrm{rad}\right)$ during time of $t_{f}=4 \mathrm{~s}$. The path of the robot is shown in figure (2):

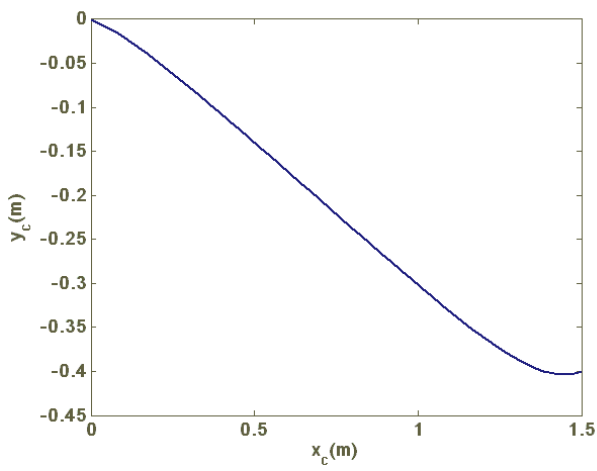

Figure 2. Path of the HILARE

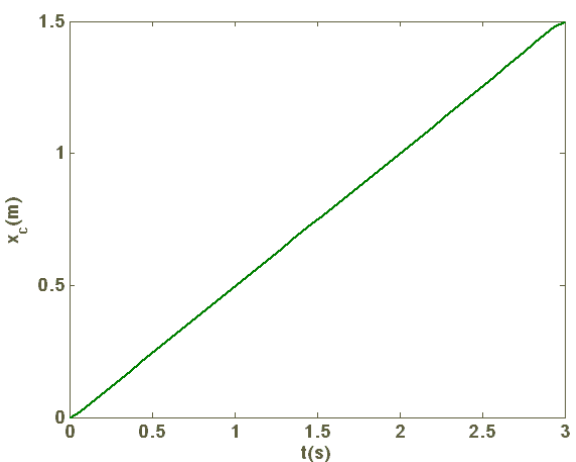

Figure 3. Horizontal displacement of the robot

As it is seen in figure (2), dynamic motion of the mobile robot is simulated regarding to derived equation of the robot. Moreover, the horizontal and vertical displacements of the robot are shown in figures (3) and (4): 


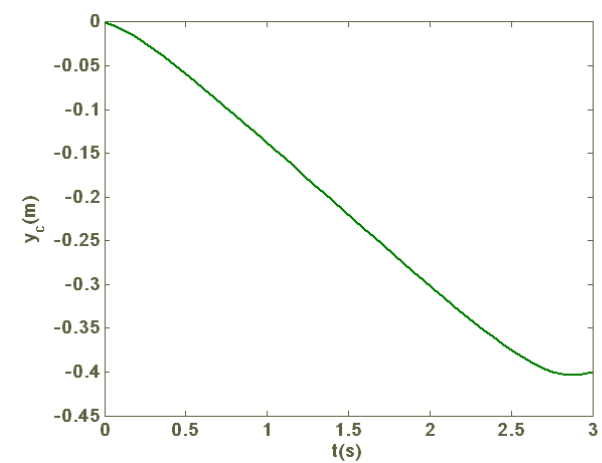

Figure 4. Vertical displacement of the robot

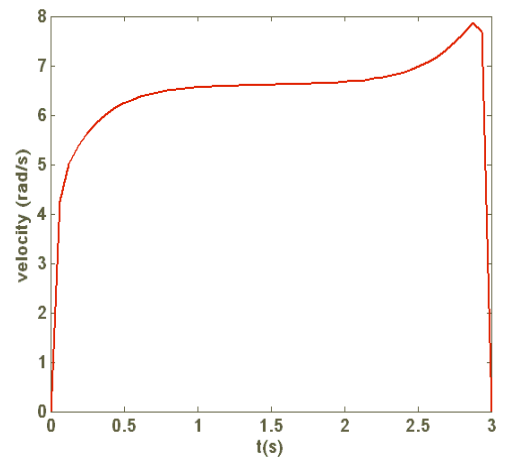

Figure 5. speed of the right fixed wheel

As it is seen in above figures, the displacement of the robot is smooth and there is not any distance between the desired and received final position of the robot. Furthermore, the velocities of the right and left fixed wheels of the robot are presented as:

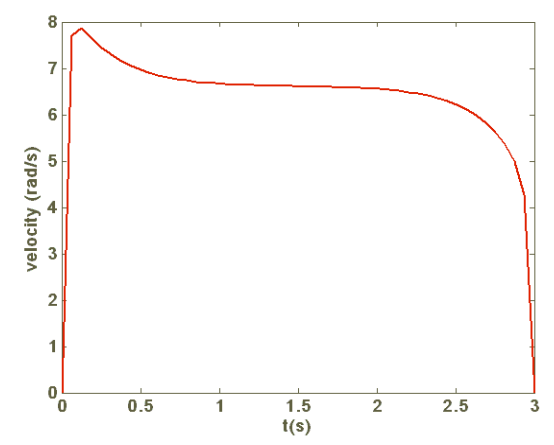

Figure 6. speed of the left fixed wheel

As it is seen in simulation study, dynamic motion of the HILARE is simulated based on derived equations of the system.

\section{CONCLUSION}

The mobile robot HILARE is a known wheeled mobile robot which has two fixed wheels and an off-entered orientable wheel. In this paper, the dynamic equations of the robot have been derived employing Lagrange multipliers method. The robot has nonholonomic constraints because the robot's wheels can only move in the direction of its plane and must have pure rolling without any slippage. Thu, the kinematic constraints of wheels have been taken into consideration and the matrix form of the kinematic model of the robot has been derived. Furthermore, dynamic model of the robot has been developed by consideration of kinematic constraints. Then, the governing equations of the robot in state-pace form have been presented. Finally, some simulations have been presented to show applicability of the proposed formulation for dynamic analysis of the mobile robot HILARE.

\section{REFERENCES}

[1] Omranpour, H., \& Shiry, S. (2012). Reduced Search Space Algorithm for Simultaneous Localization and Mapping in Mobile Robots. IAES International Journal of Robotics and Automation (IJRA), 1(1), 49-63.

[2] Behravesh, V., \& Farshchi, S. M. R. (2012). A Novel Randomized Search Technique for Multiple Mobile Robot Paths Planning In Repetitive Dynamic Environment. IAES International Journal of Robotics and Automation (IJRA), 1(4), 214-222.

[3] Korayem, M. H., Nazemizadeh, M., \& Azimirad, V. (2011). Optimal trajectory planning of wheeled mobile manipulators in cluttered environments using potential functions. Scientia Iranica, 18(5), 1138-1147.

[4] Nagaraja, H., Aswani, R., \& Malik, M. (2012). Plant Watering Autonomous Mobile Robot. IAES International Journal of Robotics and Automation (IJRA),1(3), 152-162 
[5] Rahimi, H. N., \& Nazemizadeh, M. (2014). Dynamic analysis and intelligent control techniques for flexible manipulators: a review. Advanced Robotics,28(2), 63-76

[6] Bauzil, G., Briot, M., \& Ribes, P. (1981, April). A navigation sub-system using ultrasonic sensors for the mobile robot HILARE. In 1st In.. Conf. on Robot Vision and Sensory Controls, Stratford-upon-Avon, UK (pp. 47-58)

[7] Korayem, M. H., Nazemizadeh, M., \& Rahimi, H. N. (2013). Trajectory optimization of nonholonomic mobile manipulators departing to a moving target amidst moving obstacles. Acta Mechanica, 224(5), 995-1008

[8] Galindo, C., Fernndez-Madrigal, J. A., \& González, J. (2007). Multiple abstraction hierarchies for mobile robot operation in large environments. Springer Publishing Company, Incorporated

[9] Korayem, M. H., Nazemizadeh, M., \& Nohooji, H. R. (2014). Optimal point-to-point motion planning of nonholonomic mobile robots in the presence of multiple obstacles. Journal of the Brazilian Society of Mechanical Sciences and Engineering, 36(1), 221-232

[10] Korayem, M. H., Nazemizadeh, M., \& Rahimi, H. N. (2014). Dynamic optimal payload path planning of mobile manipulators among moving obstacles. Advanced Robotics, 28(20), 1389-1402

[11] W, Chen H, Woo P Y (2000) Time optimal path planning for a wheeled mobile robot. J Robot Syst. 17: 585-591

[12] Nazemizadeh, M., Rahimi, H. N., \& Khoiy, K. A. (2012). Trajectory planning of mobile robots using indirect solution of optimal control method in generalized point-to-point task. Frontiers of Mechanical Engineering, 7(1), 23-28 